\title{
The association of patellar maltracking with infrapatellar fat pad edema and chondromalacia patella: A quantitative morphological magnetic resonance imaging analysis
}

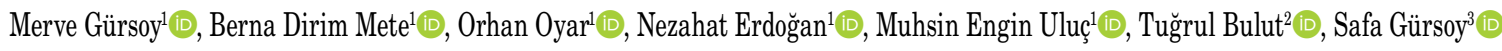 \\ ${ }^{1}$ Department of Radiology, İzmir Katip Çelebi University Atatürk Training and Research Hospital, İzmir, Turkey \\ ${ }^{2}$ Department of Orthopaedics and Traumatology, İzmir Katip Çelebi University Atatürk Training and Research Hospital, İzmir, Turkey \\ ${ }^{3}$ Department of Orthopaedics and Traumatology, Yenimahalle Training and Research Hospital, Ankara, Turkey \\ Received: April 12, 2017 Accepted: November 09, 2017 Published online: January 19, 2018
}

\begin{abstract}
Objectives: This study aims to investigate the relationship of patellofemoral joint morphology with infrapatellar fat pad edema and chondromalacia patella (CP) using patellar maltracking parameters on magnetic resonance imaging (MRI).

Patients and methods: Between January 2010 and January 2013, 50 patients with edema in the superolateral portion of the infrapatellar fat pad (the study group) and control group $(\mathrm{n}=50)$ with a normal infrapatellar fat pad were identified on MRI to compare with regard to five patellar maltracking parameters retrospectively. These parameters were trochlear depth, the trochlear sulcus angle (TSA), patellar translation, the lateral patellofemoral angle (PFA), and the Insall-Salvati ratio. The relationship between patellar maltracking and the CP was also evaluated using the same parameters.

Results: In the study group, the Insall-Salvati index and TSA were significantly higher $(\mathrm{p}=0.001)$, and the trochlear depth and PFA were low $(\mathrm{p}=0.001, \mathrm{p}=0.01)$, while patellar translation showed no difference $(\mathrm{p}=0.957)$. In the CP group, the Insall-Salvati index and TSA were significantly high $(\mathrm{p}=0.001)$, the trochlear depth was low $(\mathrm{p}=0.001)$. No statistically significant difference was found in PFA and patellar translation ( $\mathrm{p}=0.292, \mathrm{p}=0.446)$.

Conclusion: Our study results suggest that edema in the superolateral portion of infrapatellar fat pad and CP are associated with patellar maltracking.

Keywords: Chondromalacia patella; infrapatellar fat pad; magnetic resonance imaging; patellar maltracking.
\end{abstract}

The knee has three anterior fat pads; the infrapatellar fat pad, also known, as Hoffa's fat pad, the quadriceps (anterior suprapatellar) and the prefemoral (posterior suprapatellar or supratrochlear) fat pads. $^{[1]}$ The inferior pole of the patella borders the infrapatellar fat pad superiorly, joint capsule and patellar tendon borders anteriorly, proximal tibia and deep infrapatellar bursa borders inferiorly and the joint cavity borders posteriorly. ${ }^{[1]}$ Anterior extracapsular abnormalities such as patellofemoral maltracking or instability can affect this area and arise an inflammatory reaction and the edema, particularly on the superolateral part of the fat pad. It has been thought that superolateral infrapatellar fat pad edema may be associated with the friction of fat between the patellar tendon or lateral facet of patella and the lateral femoral condyle. ${ }^{[2,3]}$ This condition is called patellar tendon-lateral femoral condyle friction syndrome. ${ }^{[2]}$ Due to the lack of extensive literature on the association of patellar maltracking with infrapatellar fat pad edema, the suspicion on this view maintains. Patella alta or trochlear dysplasia, which are one of the structural abnormalities of the knee, is considered to injure the patellar cartilage because of chronic impingement between the patella and the trochlear groove during knee flexion. ${ }^{[4]}$ In the present study, we aimed to investigate the relationship of patellofemoral joint morphology with infrapatellar

Corresponding author: Merve Gürsoy, MD. İzmir Katip Çelebi Üniversitesi Atatürk Eğitim ve Araştırma Hastanesi Radyoloji Kliniği, 35360 Basın Sitesi, İzmir, Turkey. e-mail: gursoymerve@yaho0.com 
fat pad edema using different trochlear and patellar morphological parameters on magnetic resonance imaging (MRI).

\section{PATIENTS AND METHODS}

This retrospective study was approved by the Institutional Review Board of our university. The study was conducted in accordance with the principles of the Declaration of Helsinki.

Our radiology database was checked retrospectively for all patients with knee MRI between January 2010 and January 2013. Fifty patients presenting with edema in the superolateral infrapatellar fat pad (the study group) and the control group $(n=50)$ without any pathological condition in the infrapatellar fat pad were identified on MRI consecutively. Five patellar maltracking measurements were performed and two groups were compared with each other. Patients with a history of direct trauma to the knee, meniscal pathology, joint effusion, major ligament tear in the knee or patients who underwent surgery or arthroscopy were excluded.

Magnetic resonance imaging was performed on a 1.5-Tesla Signa MRI unit (General Electric Medical Systems, Milwaukee, WI, USA) with the superficial knee coil. The patient was in the neutral position.

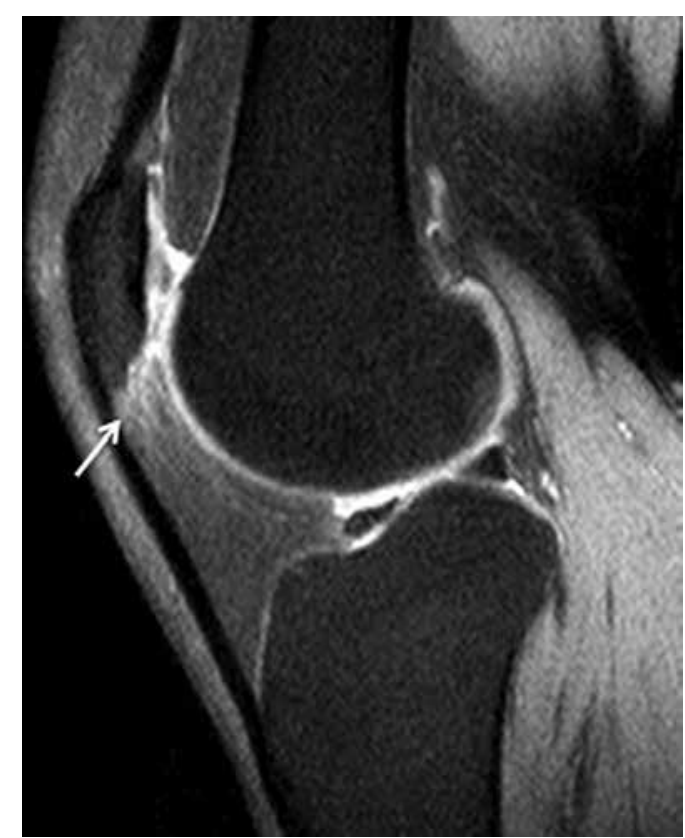

Figure 1. Sagittal proton density turbo spin-echo fat-suppressed knee magnetic resonance imaging showing high signal intensity within the superolateral aspect of the infrapatellar fat pad (arrow).
Sagittal proton-density fat-suppressed turbo spinecho (TSE) (TR/TE: 1,500/15, acquisition matrix: $256 \times 384,18 \mathrm{~cm}$ field of view), coronal proton-density fatsuppressed TSE (TR/TE: 1,500/15, acquisition matrix: $256 \times 320,18 \mathrm{~cm}$ field of view), coronal $\mathrm{T}_{1}$-weighted TSE (TR/TE: 500/18, acquisition matrix: $256 \times 320,21 \mathrm{~cm}$ field of view, two excitations), and axial $\mathrm{T}_{2}$-weighted fat-suppressed TSE (TR/TE: 2000/60, acquisition matrix: $256 \times 288,16 \mathrm{~cm}$ field of view, two excitations) sequences were included into the scan as the standard sequences. The slice thickness was $3 \mathrm{~mm}$ with a $1 \mathrm{~mm}$ interslice gap. An attending musculoskeletal radiologist blindly reviewed knee MRIs.

\section{Image analysis}

Patellar maltracking measurements (trochlear depth, the trochlear sulcus angle (TSA), patellar translation, the lateral patellofemoral angle (PFA) and the Insall-Salvati ratio) were performed on the entire knee MRI scans of the study and control groups. Fat pad edema was recorded as positive, when there was high signal intensity in the superolateral part of infrapatellar fat pad on the proton density fat-suppressed images (Figure 1).

The trochlear depth was the distance from the line connecting the most anterior parts of the medial and lateral femoral trochlea and the deepest trochlear

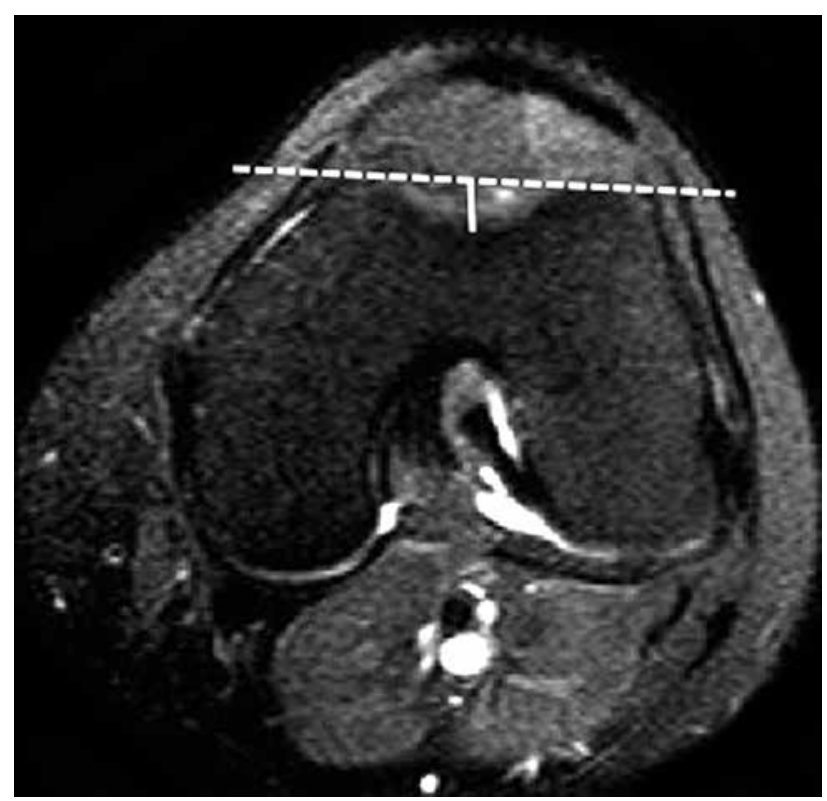

Figure 2. Trochlear depth measurement. Axial $\mathrm{T}_{2}$-weighted turbo spin-echo fat-suppressed knee magnetic resonance imaging at the level $3 \mathrm{~cm}$ above the tibiofemoral joint line. Trochlear depth is measured by vertical distance (solid line) between deepest point of sulcus and line tangential to the anterior aspect of the femoral condyles (dashed line). 
point. It was assessed on the axial slices $3 \mathrm{~cm}$ above the tibiofemoral joint line (Figure 2).

The TSA (the angle between the medial and lateral facets of the femoral trochlea with the deepest trochlear point) was measured at the level $3 \mathrm{~cm}$ above the tibiofemoral joint line on the axial slices (Figure 3).

Patellar translation was defined as the distance between the most medial point of the patella and a line drawn at the most medial point of the femoral trochlea. The line was perpendicular to the tangent drawn along the most anterior parts of the femoral trochlea) was measured at the most medial part of the patella level on the axial images (Figure 4).

The PFA (the angle between the line drawn along the bony lateral patellar facet and another line drawn tangential to the anterior point of the femoral condyles) was assessed at the mid-point of the patella on the axial images (Figure 5).

The Insall-Salvati ratio (a ratio between the patellar tendon length and the patellar diagonal height) was measured at the greatest length of patella level on sagittal images (Figure 6). The two groups were also compared with regard to the Insall-Salvati index with cut-off values. An Insall-Salvati index, which was more than 1.3 , was defined as patella alta.

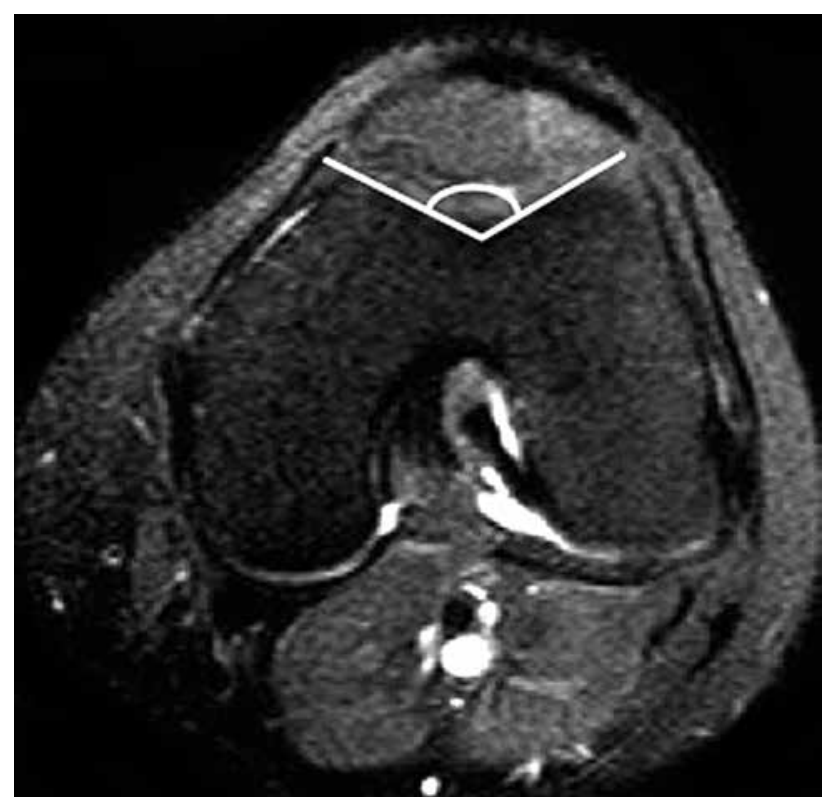

Figure 3. Trochlear sulcus angle measurement. Axial $\mathrm{T}_{2}$-weighted turbo spin-echo fat-suppressed knee magnetic resonance imaging at the level $3 \mathrm{~cm}$ above the tibiofemoral joint line. Trochlear sulcus angle is measured between lines paralleling bony cortex of femoral trochlea.
Magnetic resonance imaging scans were also reviewed to detect the absence or presence of chondromalacia patellae.

Patellar cartilage was assessed for focal signal or focal contour abnormality on either the $\mathrm{T}_{2}$-weighted or proton density-weighted images. The patients with chondromalacia patellae (without considering the grades) and without chondromalacia patellae were evaluated using the same five patellar maltracking parameters, and the relationship between patellofemoral joint morphology and the presence of chondromalacia patellae was interpreted.

\section{Statistical analysis}

Statistical analysis was performed using SPSS, version 15.0 (SPSS Inc., Chicago, IL, USA). The trochlear depth, TSA, patellar translation, PFA, and the Insall-Salvati ratio values were compared using the unpaired Student's t-test between the patients with superolateral infrapatellar fat pad edema and control group. The Student's t-test was also used to compare the same patellar maltracking parameters between the group with chondromalacia patellae and without chondromalacia patellae. The alpha level of statistical significance was set at 0.05 . G ${ }^{\star}$ Power 3.1 software

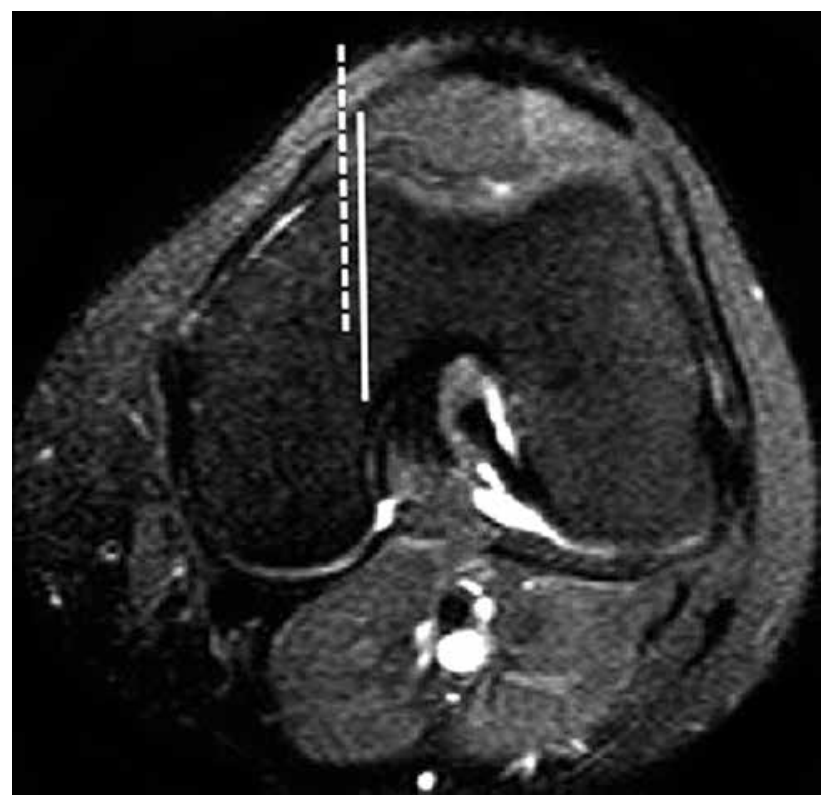

Figure 4. Patellar translation measurement. Axial $\mathrm{T}_{2}$-weighted turbo spin-echo fat-suppressed knee magnetic resonance imaging image at the level $3 \mathrm{~cm}$ above the tibiofemoral joint line. Distance between parallel lines from medial edge of patella (dashed line) through most anterior point of medial condyle (solid line). Lines are perpendicular to tangent line through posterior femoral condyles. 


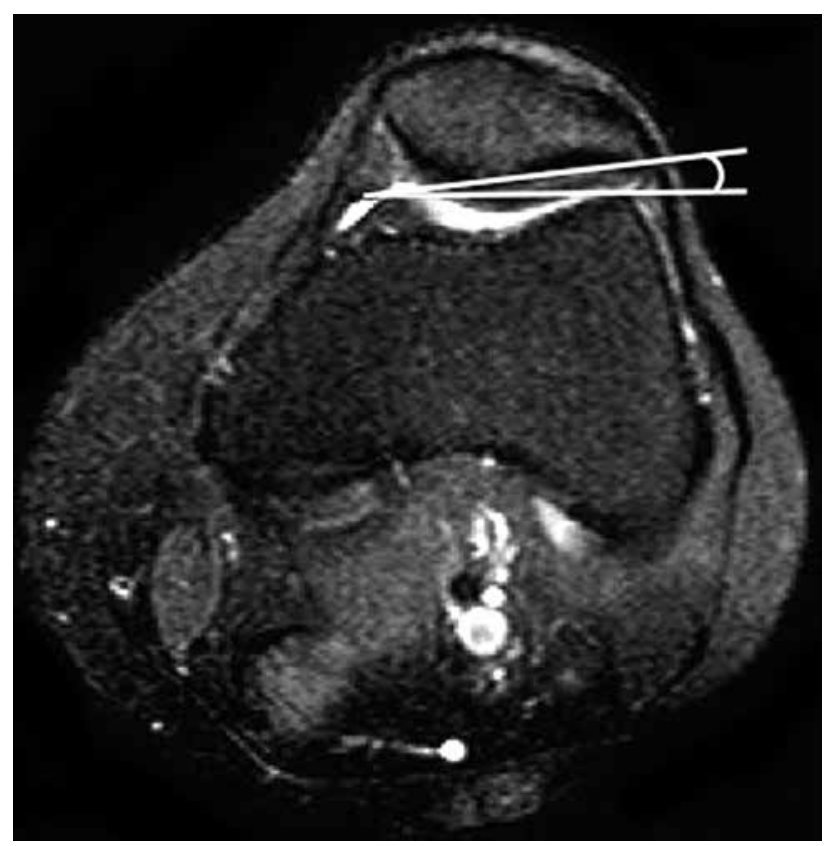

Figure 5. Patellofemoral angle measurement at the midpoint of the patella. Axial $\mathrm{T}_{2}$-weighted turbo spin-echo fat-suppressed knee magnetic resonance imaging shows the angle formed by a line drawn along the bony lateral patellar facet and another line drawn tangential to the anterior aspect of the femoral condyles.

program (Heinrich Heine University, Dusseldorf, Germany) was used to determine the sample size. When the effect size was taken 0.50 and power 0.95 , total sample size was calculated as 42 for the continuous variables and 52 for categorical variables.

\section{RESULTS}

The study group consisted of 50 patients with superolateral infrapatellar fat pad edema, 33 females (66\%) and 17 males (34\%) (mean age 38.6 \pm 12.1 years; range, 19 to 66 years). The control group consisted of 50 patients without edema, 22 females (44\%) and 28 males (56\%) (mean age 26.3 \pm 7.3 years; range $18-43$ years). Age distribution was higher in the study group $(\mathrm{p}<0.001)$.

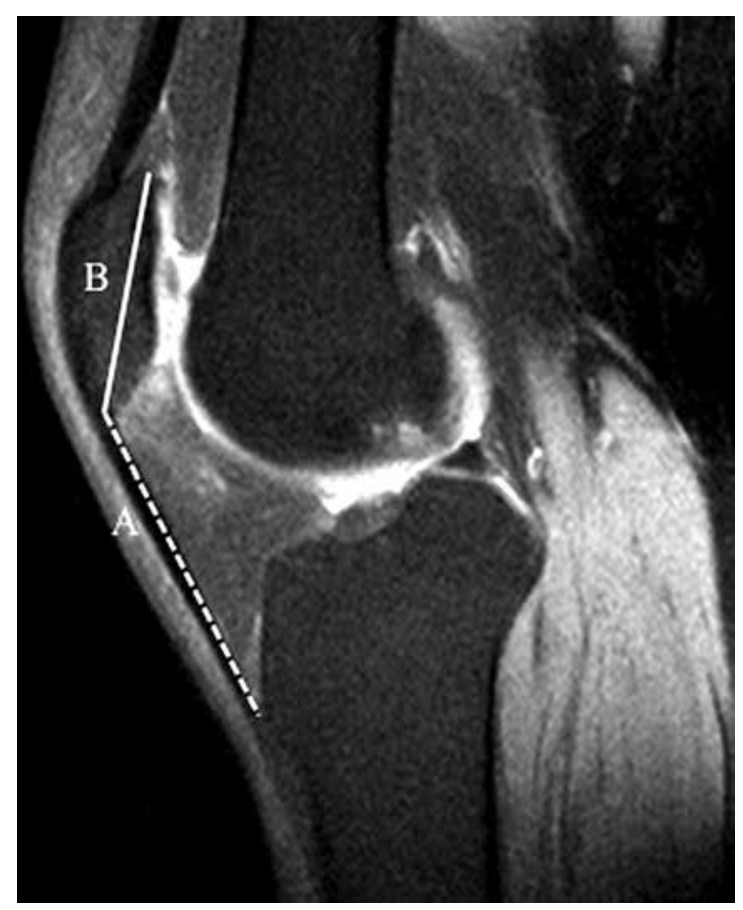

Figure 6. The Insall-Salvati ratio measurement on the sagittal proton density turbo spin-echo fat-suppressed knee magnetic resonance imaging. The ratio=A/B. A: Distance of patellar tendon from inferior patellar pole to tibial tubercle (dashed line). B: Greatest diagonal length of patella (solid line).

The groups were compared with regard to five patellar maltracking measurements. The Insall-Salvati index and TSA values were significantly higher in the study group $(\mathrm{p}=0.001)$. The trochlear depth and PFA were significantly lower in the study group $(\mathrm{p}=0.001, \mathrm{p}=0.01)$. With regard to patellar translation, we found no significant difference between two groups $(\mathrm{p}=0.957)$ (Table 1). When the cut-off of 1.3 was taken, the frequency of patella alta was significantly higher in the study group ( $\mathrm{p}=0.001$, in the study group in $29 / 50$ patients (58\%) and in the control group in $10 / 50$ patients (20\%).

The other objective of this study was to compare the patients with chondromalacia patellae (chondromalacia

Table 1. Mean values of patellar maltracking parameters in the study and the control groups

\begin{tabular}{|c|c|c|c|}
\hline & Study group $(\mathrm{n}=50)$ & Control group $(\mathrm{n}=50)$ & $p$ \\
\hline & Mean \pm SD & Mean \pm SD & \\
\hline Trochlear depth (mm) & $3.2 \pm 1.1$ & $4.6 \pm 1.3$ & 0.001 \\
\hline Trochlear sulcus angle & $148.2 \pm 10.9$ & $134.6 \pm 8.6$ & 0.001 \\
\hline Lateral patellofemoral angle & $9.3 \pm 3.7$ & $11.1 \pm 4.0$ & 0.01 \\
\hline Patellar translation & $2.8 \pm 1.4$ & $2.8 \pm 1.4$ & 0.957 \\
\hline Insall-Salvati index & $1.4 \pm 0.2$ & $1.1 \pm 0.2$ & 0.001 \\
\hline
\end{tabular}


Table 2. Mean values of patellar maltracking parameters in the chondromalacia and the normal groups

\begin{tabular}{lcccc}
\hline & Chondromalasia group $(\mathrm{n}=50)$ & & Normal group $(\mathrm{n}=50)$ & $p$ \\
\cline { 5 - 5 } & Mean \pm SD & & Mean \pm SD & \\
\hline Trochlear depth & $3.4 \pm 1.0$ & $4.6 \pm 1.3$ & 0.001 \\
Trochlear sulcus angle & $146.4 \pm 10.1$ & $134.6 \pm 8.6$ & 0.001 \\
Lateral patellofemoral angle & $10.1 \pm 3.5$ & $11.1 \pm 4.0$ & 0.292 \\
Patellar translation (mm) & $2.5 \pm 1.1$ & $2.8 \pm 1.4$ & 0.446 \\
Insall-Salvati index & $1.4 \pm 0.2$ & $1.1 \pm 0.2$ & 0.001 \\
\hline SD: Standard deviation. & & &
\end{tabular}

group) and the patients with a normal cartilage (normal group), whether there was a significance difference in the distribution of five patellar maltracking measurements. The chondromalacia group consisted of 30 patients, 14 females (47\%) and 16 males (53\%) (mean age: $31 \pm 10.4$ years, range: 25 to 71 years). The control group consisted of 50 patients without edema, 22 females (44\%) and 28 males (56\%) (mean age $26.3 \pm 7.3$ years; range, 18 to 43 years). Age distribution was higher in the chondromalacia group $(p=0.01)$.

The relationship between patellofemoral maltracking and the presence of chondromalacia patellae was evaluated. There was chondromalacia patella in 30 of 50 patients $(60 \%)$. The chondromalacia group consisted of 30 patients and the normal group consisted of 50 patients. The Insall-Salvati index and TSA values were significantly higher in the chondromalacia group $(\mathrm{p}=0.001)$. The trochlear depth values of chondromalacia group were significantly lower $(p=0.001)$. There was no statistically significant difference in the PFA and patellar translation between groups with and without chondromalacia $(\mathrm{p}=0.292$, $\mathrm{p}=0.446)$ (Table 2).

\section{DISCUSSION}

In this study, we found a relationship between superolateral infrapatellar fat pad edema and patellar maltracking. Similarly, in a study by Jibri et al. ${ }^{[5]}$ including patients with and without edema in the superolateral of the infrapatellar fat pad, measurements were taken on MRI images and it was aimed to reveal any patellar instability and the relationship of that with edema. Similarly, in the current study, the InsallSalvati index values of the patient group were found to be statistically significantly higher and the PFA values were significantly lower. No difference was found in the current study between the groups in respect of patellar translation. In the study by Jibri et al., ${ }^{[5]}$ the patellar translation values of the patient group were found to be significantly higher. The tibial tuberosity-trochlear groove distance (TTTG) corresponding to the clinically measured $\mathrm{Q}$ angle, which was not used in our study, is accepted as abnormal at values of $20-\mathrm{mm}$ and over and has almost always been related to the patellar instability. Although not of a statistically significant level, Jibri et al. ${ }^{[5]}$ found the TTTG of the patient group to be higher. Similar to our study, Jibri et al..$^{[5]}$ demonstrated that edema in the superolateral portion of Hoffa's fat pad, the MRI feature of fat pad impingement, was associated with patellar maltracking.

Patellofemoral maltracking is one of the most common causes of infrapatellar fat pad impingement. Patellofemoral maltracking may be seen as the result of anatomic variations of the structures stabilizing the patellofemoral joint. The most important variations causing patellofemoral maltracking are trochlear dysplasia, patella alta and lateralization of the tibial tuberosity. Magnetic resonance imaging is an imaging method with high sensitivity, which can be used in the visualization of these anatomic variations. ${ }^{[6,7]}$

Patella alta (the patella is abnormally elevated) is the most consistent anatomic feature of patellar instability. In patella alta, when there is slight flexion it is not possible for the patellar bone to sit completely in the trochlear groove. It has been suggested that in cases of patella alta, the patellofemoral contact area is on average $19 \%$ less than normal. ${ }^{[8]}$ These factors are likely related with articular cartilage abnormalities as well as patellar instability in patients with patella alta. In the current study, there was a significant difference between the two groups in respect of the frequency of patella alta, which was found in the patient group in $29 / 50$ patients $(58 \%)$ and in the control group in $10 / 50$ patients $(20 \%)(\mathrm{p}=0.001)$.

In trochlear dysplasia, the trochlear groove is flattened in the proximal and the concavity is reduced in the distal. Due to these changes, during extension and early flexion movements, there is a tendency for patella lateral subluxation. Trochlear depth and TSA have been used as markers for the presence of trochlear dysplasia. In previous studies, TSA $>144^{\circ}$ has been 
accepted as an indicator of hypoplasia. ${ }^{[9]}$ Trochlear depth of $>5 \mathrm{~mm}$ has been accepted as hypoplasia and $<3 \mathrm{~mm}$ as an indicator of dysplasia. ${ }^{[6]}$ The prevalence of trochlear dysplasia was significantly higher in the study group. In addition, TSA was significantly higher and the trochlear depth was lower in the study group. Trochlear dysplasia may also impair the patellofemoral joint stability and cause patellar instability, patellar dislocation, and finally softening or loss of patellofemoral cartilage. ${ }^{[10]}$ This condition results from chronic friction between the patella and the trochlear groove through which it passes during knee flexion. Although there are few studies in the literature, which have related chondromalacia patella with trochlear dysplasia, the results are conflicting. While Endo et al. ${ }^{[4]}$ did not find a relationship between the depth of trochlea and chondromalacia patellae, Ali et al. ${ }^{[1]}$ reported that severe cartilage defects were associated with both the trochlear depth and the sulcus angle. In our study, similar to Ali et al. ${ }^{[1]}$ the trochlear depth was significantly lower and TSA was significantly higher in the chondromalacia group.

In the literature, there have been numerous studies addressing into the Insall-Salvati index and its relationship with chondromalacia patellae. ${ }^{[12-14]}$ Abnormally elevated patella leads to the incongruity between the patellar cartilage and trochlea. In our study, the Insall-Salvati index of the chondromalacia group was significantly higher $(p=0.001)$. On the contrary, Endo et al.$^{[4]}$ found no statistically significant difference in the ratio of patellar tendon length to patellar length between the knees with and those without chondromalacia patellae.

Tuna et al. ${ }^{[15]}$ demonstrated a significant correlation between the lateral patellar tilt with chondromalacia patellae on MRI. Those who found a relationship between the PFA and chondromalacia patellae reasoned that when the PFA decreases, the lateral inclination of patella increases and this condition impairs the lateral stabilizing mechanism of patella. Our study was the second that investigated the association of PFA with chondromalacia patellae on MRI. However, there was no statistically significant difference in PFA, as well as patellar translation between groups with and without chondromalacia.

There are some limitations of this study. It is retrospective and the radiologist who made the measurements was aware of the presence or absence of fat pad edema. This situation might have been caused a bias. Another limitation is that we diagnosed the chondromalacia patella on MRI, but we were unable to correlate it to arthroscopic findings. Therefore, normal cartilage observed on MRI might have been early stage of chondromalacia at arthroscopy. Finally, due to the retrospective nature of the study, the standard sequences were obtained for the review. Cartilage-specific three-dimensional MRI techniques that provide detailed information of the cartilage might have revealed obscured mild cartilage lesions.

In conclusion, concerning morphology of patellofemoral joint, we demonstrated an association between the abnormality of femoral trochlear osseous anatomy, the position of patella and infrapatellar fat pad edema. We also demonstrated a significant relationship between patellar maltracking and chondromalacia patella. Significantly higher sulcus angle, lower trochlear depth and abnormally elevated patella were seen in patients with chondromalacia patella. The infrapatellar fat pad impingement is one of the causes of anterior knee pain. To investigate the relationship between patellar maltracking and infrapatellar fat pad edema may help to find out the underlying cause of patient symptoms and this might change the treatment method. Therefore, a radiologist should be aware of this entity to guide the clinician to the right treatment strategy.

\section{Declaration of conflicting interests}

The authors declared no conflicts of interest with respect to the authorship and/or publication of this article.

\section{Funding}

The authors received no financial support for the research and/or authorship of this article.

\section{REFERENCES}

1. Jacobson JA, Lenchik L, Ruhoy MK, Schweitzer ME, Resnick D. MR imaging of the infrapatellar fat pad of Hoffa. Radiographics 1997;17:675-91.

2. Chung CB, Skaf A, Roger B, Campos J, Stump X, Resnick D. Patellar tendon-lateral femoral condyle friction syndrome: MR imaging in 42 patients. Skeletal Radiol 2001;30:694-7.

3. Subhawong TK, Eng J, Carrino JA, Chhabra A. Superolateral Hoffa's fat pad edema: association with patellofemoral maltracking and impingement. AJR Am J Roentgenol 2010;195:1367-73.

4. Endo Y, Schweitzer ME, Bordalo-Rodrigues M, Rokito AS, Babb JS. MRI quantitative morphologic analysis of patellofemoral region: lack of correlation with chondromalacia patellae at surgery. AJR Am J Roentgenol 2007;189:1165-8.

5. Jibri Z, Martin D, Mansour R, Kamath S. The association of infrapatellar fat pad oedema with patellar maltracking: a case-control study. Skeletal Radiol 2012;41:925-31. 
6. Chhabra A, Subhawong TK, Carrino JA. A systematised MRI approach to evaluating the patellofemoral joint. Skeletal Radiol 2011;40:375-87.

7. Diederichs G, Issever AS, Scheffler S. MR imaging of patellar instability: injury patterns and assessment of risk factors. Radiographics 2010;30:961-81.

8. Ward SR, Terk MR, Powers CM. Patella alta: association with patellofemoral alignment and changes in contact area during weight-bearing. J Bone Joint Surg Am 2007;89:1749-55.

9. Salzmann GM, Weber TS, Spang JT, Imhoff AB, Schöttle $\mathrm{PB}$. Comparison of native axial radiographs with axial MR imaging for determination of the trochlear morphology in patients with trochlear dysplasia. Arch Orthop Trauma Surg 2010;130:335-40.

10. Rebolledo BJ, Nam D, Cross MB, Green DW, Sculco TP. Familial association of femoral trochlear dysplasia with recurrent bilateral patellar dislocation. Orthopedics
2012;35:574-9.

11. Ali SA, Helmer R, Terk MR. Analysis of the patellofemoral region on MRI: association of abnormal trochlear morphology with severe cartilage defects. AJR Am J Roentgenol 2010;194:721-7.

12. Dowd GS, Bentley G. Radiographic assessment in patellar instability and chondromalacia patellae. J Bone Joint Surg Br 1986;68:297-300.

13. Lancourt JE, Cristini JA. Patella alta and patella infera. Their etiological role in patellar dislocation, chondromalacia, and apophysitis of the tibial tubercle. J Bone Joint Surg Am 1975;57:1112-5.

14. Marks KE, Bentley G. Patella alta and chondromalacia. J Bone Joint Surg Br 1978;60:71-3.

15. Tuna BK, Semiz-Oysu A, Pekar B, Bukte Y, Hayirlioglu A. The association of patellofemoral joint morphology with chondromalacia patella: a quantitative MRI analysis. Clin Imaging 2014;38:495-498. 